\title{
Maintaining the balance: Another responsibility of a journal and its editor
}

An editor and editorial board of a serious plastic surgical journal have the responsibilities of encouraging, seeking, preparing and publishing articles of excellence, importance and relevance. These obligations are crucial to the wellbeing of the publisher, the authors, the readers and ultimately patients. A more subtle yet critical responsibility is to achieve a balance in its content. For our specialty this means giving due consideration and prominence to aesthetic, reconstructive and research components, the basic assumption being that the Editor receives enough articles from all these areas. In that regard, when my predecessor, Dr. Frank McDowell, first became Editor of Plastic and Reconstructive Surgery in 1969, he did not receive sufficient submissions to have the dilemma and luxury of selecting from a plethora of manuscripts. When I succeeded Dr. McDowell in 1979 and increasingly during my tenure until 2005, the influx of articles increased as did the rate of rejection. For me the most difficult, albeit necessary, aspect of being Editor was sending an author a letter of rejection, which over the many years of being an author; I also received with its attendant unpleasantness.

A good editor should never let a desire for popularity overcome his or her responsibility to be the final sieve between author and reader. Being an editor or a member of the editorial board or a reviewer entails the discomfort of having to make difficult decisions. After all, as surgeons we face these situations in our daily work.

As editor of Plastic and Reconstructive Surgery, I tried, hopefully with some success, to keep in mind the needs of readers and their patients. That I was criticized from time to time for publishing too many reconstructive and experimental articles and not enough aesthetic was a reality I was willing to accept because of the diversity of our readership, with differing needs and expectations. Our specialty has a wider spectrum than the surgery of appearance or what is becoming popularly known as cosmetic medical care, e.g., injection of botox and fillers.
An international journal of stature, like this one, especially based in India, must impart to its readers sufficient knowledge to help patients whose problems are restoration of function, elimination of cancer, reconstruction of defects and even survival. For this reason, a judicious Editor, whom this Journal fortunately has, must maintain a balance, as Dr. Mukund Thatte intends.$^{[1]}$ Only in this way can a publication become and remain a vital instrument for educating its readers and aiding their patients. This objective necessitates airing not just clinical advances in all of plastic surgery but experimental research. This is required particularly in our specialty whose practitioners, as they age, become further distant from basic science. What happens in the laboratory today will likely be evident in practice in the future, but not necessarily tomorrow, next month or even next year. A journal such as this must reflect not only what is happening in plastic surgery but its possible future directions to stimulate its readers to ponder and investigate.

What makes our specialty of plastic surgery so interesting is the variety of the problems we confront. It is mandatory that we have a diversity of knowledge and its application. The editorial problems and challenges arising from striving to keep the balance within the spectrum of plastic surgery, even from other fields of endeavor and educating all readers are far outweighed by the positive ramifications, extending to the patients whom we serve and who should be our most important concern.

Robert M. Goldwyn

Clinical Professor of Surgery, Harvard Medical School, Editor Emeritus, Plastic and Reconstructive Surgery, 54 Willow Crescent, Brookline, Massachusetts 02445, USA. E-mail: tatyanarfa@comcast.net

\section{REFERENCE}

1. Thatte M. Musings at the year end. Indian J Plast Surg 2006;39:125 